\title{
Moving into the next era of PET myocardial perfusion imaging: introduction of novel ${ }^{18} \mathrm{~F}$-labeled tracers
}

\author{
Rudolf A. Werner ${ }^{1,2,3} \cdot$ Xinyu Chen $^{2,3} \cdot$ Steven P. Rowe ${ }^{1} \cdot$ Constantin Lapa ${ }^{2} \cdot$ Mehrbod S. Javadi $^{1} \cdot$ Takahiro Higuchi $^{2,3,4}$
}

Received: 20 August 2018 / Accepted: 12 October 2018 / Published online: 17 October 2018

(c) The Author(s) 2018

\begin{abstract}
The heart failure epidemic continues to rise with coronary artery disease as one of its main causes. Novel concepts for risk stratification to guide the referring cardiologist towards revascularization procedures are of significant value. Myocardial perfusion imaging using single-photon emission computed tomography (SPECT) agents has demonstrated high accuracy for the detection of clinically relevant stenoses. With positron emission tomography (PET) becoming more widely available, mainly due to its diagnostic performance in oncology, perfusion imaging with that modality is more practical than in the past and overcomes existing limitations of SPECT MPI. Advantages of PET include more reliable quantification of absolute myocardial blood flow, the routine use of computed tomography for attenuation correction, a higher spatiotemporal resolution and a higher count sensitivity. Current PET radiotracers such as rubidium-82 (half-life, $76 \mathrm{~s}$ ), oxygen-15 water (2 min) or nitrogen-13 ammonia (10 min) are labeled with radionuclides with very short half-lives, necessitating that stress imaging is performed under pharmacological vasodilator stress instead of exercise testing. However, with the introduction of novel ${ }^{18} \mathrm{~F}$-labeled MPI PET radiotracers (half-life, $110 \mathrm{~min}$ ), the intrinsic advantages of PET can be combined with exercise testing. Additional advantages of those radiotracers include, but are not limited to: potentially improved cost-effectiveness due to the use of pre-existing delivery systems and superior imaging qualities, mainly due to the shortest positron range among available PET MPI probes. In the present review, widely used PET MPI radiotracers will be reviewed and potential novel ${ }^{18} \mathrm{~F}$-labeled perfusion radiotracers will be discussed.
\end{abstract}

Keywords Coronary artery disease $\cdot$ Precision medicine $\cdot$ Positron emission tomography $\cdot$ Myocardial perfusion imaging $\cdot$ ${ }^{18} \mathrm{~F}$-flurpiridaz $\cdot{ }^{18} \mathrm{~F}$-FBnTP

Electronic supplementary material The online version of this article (https://doi.org/10.1007/s10554-018-1469-z) contains supplementary material, which is available to authorized users.

Takahiro Higuchi

thiguchi@me.com

1 Division of Nuclear Medicine and Molecular Imaging, The Russell H. Morgan Department of Radiology and Radiological Science, Johns Hopkins University School of Medicine, Baltimore, MD, USA

2 Department of Nuclear Medicine, University of Wuerzburg, Wuerzburg, Germany

3 Comprehensive Heart Failure Center, University of Wuerzburg, Oberduerrbacher Strasse 6, 97080 Wuerzburg, Germany

4 Department of Biomedical Imaging, National Cardiovascular and Cerebral Center, Suita, Japan

\section{Introduction}

The heart failure (HF) epidemic continues to rise with an estimated future financial burden of $\$ 70$ billion in the year $2030[1,2]$. Notably, HF has been recently further subclassified into HF with reduced ejection fraction (HFrEF), with preserved ejection fraction (HFpEF), and an intermediate group (HF with mid-range ejection fraction, $\mathrm{HFmrEF}$ ) [3, 4]. However, one of the main characteristics of either HFrEF, HFpEF or HFmrEF is coronary artery disease (CAD, in up to $54 \%$ of the cases) $[3,5,6]$ and therefore, its reliable detection, preferably at an early stage of disease, is as relevant as ever [7]. As a result of these considerations, novel strategies for the assessment of flow-limiting coronary artery stenoses have been extensively investigated and myocardial perfusion imaging (MPI) has been an important part of evaluating for this pathology. The most commonly used radiotracers for MPI are the single-photon emission computed tomography 
(SPECT) agents ${ }^{99 \mathrm{~m}} \mathrm{Tc}$-labeled sestamibi and tetrofosmin, as well as thallium-201 $\left({ }^{201} \mathrm{TI}\right)$ [8]. In general, the use of positron emission tomography (PET) is expanding worldwide, mainly due to its superior diagnostic performance in oncology [9, 10]. Thus, MPI may benefit from the increasing installed base of latter imaging modality, as PET may provide advantages over SPECT MPI imaging. First, PET has a higher spatiotemporal resolution in comparison to SPECT and a higher count sensitivity. In this light, several studies have already reported on the superior imaging characteristics and higher accuracy of PET MPI compared to conventional SPECT MPI [11, 12]. Moreover, PET includes attenuation correction on a routine basis, as hybrid systems equipped with computed tomography (CT) are routinely installed, which also allows for anatomic co-registration [13]. Apart from that, with traditional PET agents, both rest and stress images can be acquired during one single study, mainly due to the shorter half-life of PET agents [14] and PET has also opened the door for reliable quantification of absolute myocardial blood flow (MBF) $[15,16]$.

Nonetheless, expensive production procedures with onsite cyclotrons are needed for short-half-life agents [14]. This is in contradistinction to recent developments of novel ${ }^{18} \mathrm{~F}$-labeled radiotracers, which may overcome some of the hurdles to adoption of established PET MPI agents. First, ${ }^{18}$ F-labeled imaging probes for MPI may be distributed using delivery systems from central cyclotron facilities. Second, the longer half-life of ${ }^{18} \mathrm{~F}$-labeled MPI agents also allows for delayed imaging protocols. From a practical standpoint, exercise stress testing outside of the scanner is feasible [17]. This manuscript reviews this novel class of PET radiotracers for MPI. Among those, ${ }^{18} \mathrm{~F}$-flurpiridaz (also previously referred as ${ }^{18} \mathrm{~F}$-BMS747158-02) and ${ }^{18} \mathrm{~F}$-fluorobenzyltriphenyl-phosphonium ( $\left.{ }^{18} \mathrm{~F}-\mathrm{FBnTP}\right)$ have been extensively evaluated and thus, will be further discussed.

\section{Clinical PET radiotracers for MPI and advantages of ${ }^{18} \mathrm{~F}$-labeled radiotracers}

To date, the clinically used PET MPI agents are rubidium 82 $\left({ }^{82} \mathrm{Rb}\right.$, half-life, $\left.76 \mathrm{~s}\right)$, oxygen-15-water $\left({ }^{15} \mathrm{O}\right.$-water, half-life $2 \mathrm{~min})$ and nitrogen-13-ammonia $\left({ }^{13} \mathrm{~N}\right.$-ammonia, half-life, $10 \mathrm{~min}$ ) [18]. For the production of ${ }^{82} \mathrm{Rb}$, a commercially available strontium 82 generator is needed, and the high cost for a monthly replacement $(\$ 20,000)$ is a consideration for practitioners as to what extent ${ }^{82} \mathrm{Rb}$ PET MPI can be employed in clinical routine [17]. Further drawbacks include its ultrashort half-life and the lowest first-pass extraction (65\%) among all available PET MPI agents. In addition, the maximum kinetic energy of positrons emitted during ${ }^{82} \mathrm{Rb}$ decay is much higher than that of ${ }^{13} \mathrm{~N}$ and ${ }^{18} \mathrm{~F}$ [19]. The latter aspect may have an impact on image quality: high-energy positrons have a long average distance to annihilation and, therefore, the spatial resolution is lower relative to other radionuclides with lower positron energies [17]. The production of ${ }^{15} \mathrm{O}$-water PET depends on a cyclotron unit and it is seen as the gold standard for flow quantification, as it freely diffuses across the cardiomyocyte membrane and produces ideal flow measurements [20]. However, its noisy low-count imaging quality as well as necessity of complex kinetic modeling limits its clinical use [18]. ${ }^{13} \mathrm{~N}$-ammonia is approved by the United States Food and Drug Administration and has a very good image quality, but it also requires a costly on-site cyclotron [18, 21].

Notably, use of ${ }^{18} \mathrm{~F}$ radionuclides may overcome these limitations of commonly used PET MPI radiotracers. Advantages of ${ }^{18} \mathrm{~F}$ as a radionuclide include, but are not limited to:

(I) ${ }^{18} \mathrm{~F}$ has a relatively long physical half-life of $110 \mathrm{~min}$, which allows for the use of delivery systems [22] and such an approach has already been proven to be costeffective for 2-deoxy-2- ${ }^{18} \mathrm{~F}$-fluoro-D-glucose $\left({ }^{18} \mathrm{~F}-\mathrm{FDG}\right)$ [23];

(II) ${ }^{18} \mathrm{~F}$ has the shortest positron range in tissue compared to other established MPI PET radionuclides [19], and, thus, it may have the highest spatial resolution [17];

(III) the lower positron energy with higher positron yield allows for injection of a considerably lower amount of radioactivity [13];

(IV) its long half-life opens the door for delayed imaging protocols (e.g. for assessment of blood flow alterations at late scan time-points) [24];

(V) due to the short half-life of currently used PET MPI agents, stress imaging is only feasible while placing the patient under pharmacological stress. Notably, ${ }^{18}$ F-labeled radiotracers may overcome this limitation by allowing for physical exercise stress testing outside of the PET device $[17,25,26]$.

To date, the most extensively studied ${ }^{18} \mathrm{~F}$-labeled radiotracer for PET MPI is ${ }^{18} \mathrm{~F}$-flurpiridaz (Fig. 1).

\section{${ }^{18} \mathrm{~F}$-labeled radiotracers for MPI: ${ }^{18} \mathrm{~F}$-flurpiridaz}

\section{Preclinical evaluation}

${ }^{18} \mathrm{~F}$-flurpiridaz has demonstrated favorable imaging characteristics for MPI in preclinical studies: As a derivative of the pyridazinone insecticide pyridaben, it has a high binding affinity towards mitochondrial complex I, with a considerable high first-pass extraction of $>90 \%$ as measured in an isolated perfused heart setup [27, 28]. Comparing ${ }^{18} \mathrm{~F}$-flurpiridaz with the established SPECT agent 
Fig. 1 Overview of the herein reviewed ${ }^{18} \mathrm{~F}$-labeled PET radiotracers for MPI, namely ${ }^{18} \mathrm{~F}$-flurpiridaz and ${ }^{18}$ F-fluorobenzyltriphenyl-phosphonium $\left({ }^{18} \mathrm{~F}-\mathrm{FBnTP}\right)$<smiles>CC(C)(C)n1ncc(OCc2ccc(COCC[18F])cc2)c(Cl)c1=O</smiles>

${ }^{18}$ F-Flurpiridaz

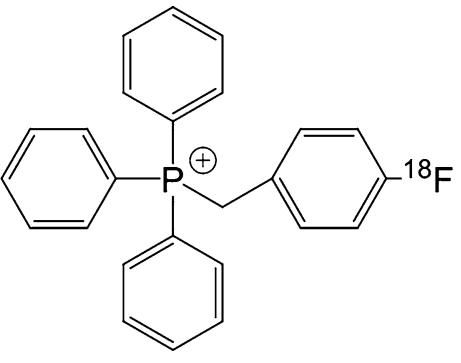

${ }^{18} \mathrm{~F}-\mathrm{FBnTP}$
${ }^{99 m}$ Tc sestamibi in a biodistribution rat study, the cardiac uptake of the ${ }^{18} \mathrm{~F}$-labeled agent was significantly higher at both early $(15 \mathrm{~min})$ and late time points $(120 \mathrm{~min})$. This experiment was followed by an isolated rabbit heart perfusion study and net ${ }^{18} \mathrm{~F}$-flurpiridaz cardiac uptake increased to a greater extent than that of ${ }^{201} \mathrm{TI}$ or ${ }^{99 \mathrm{~m}} \mathrm{Tc}$ sestamibi at physiologically relevant flow rates. Moreover, an in vivo PET study demonstrated almost no lung uptake and rapid liver clearance in rats, rabbits, and primates (pronounced washout between 5 and $15 \mathrm{~min}$ ). In addition, a rat model of coronary occlusion also showed an excellent correlation with ${ }^{18} \mathrm{~F}$-flurpiridaz uptake and histopathological findings [29]. These findings were further corroborated in a chronic myocardial infarction (MI) model in rabbits (left coronary artery occlusion, followed by recovery phase over 1 month): compared to controls, a clear defect could be appreciated in the left ventricular wall. The promising safety profile of this imaging agent was further confirmed by electrocardiogram assessments in both controls and MI rabbits [30]. Huisman et al. also used the Langendorff method and investigated the first-pass extraction of ${ }^{18} \mathrm{~F}$-flurpiridaz in isolated perfused rat hearts, on which the radiotracer demonstrated a high and flow-independent myocardial first-pass extraction fraction. Thus, ${ }^{18} \mathrm{~F}$-flurpiridaz may hold the promise of a linear correlation between radiotracer uptake and cardiac blood flow [28]. Higuchi and coworkers tested ${ }^{18}$ F-flurpiridaz in rats in vivo. Normal healthy control rats were found to have a homogoneous delineation of the myocardium up to $2 \mathrm{~h}$ after tracer injection. However, for the permanent occlusion model, the defect size remained stable over the entire imaging protocol (15-115 min). This was in contradistinction to the transient ischemia model: reperfusion after short, transient ischemia of 3 min showed radiotracer redistribution to the induced defect (i.e. tracer redistribution after reperfusion). Radiotracer reinjection further enhanced the normalization process. The concept of redistribution is based on underperfused but viable myocardium, which retains the radiotracer while it washes out of normal myocardial areas, i.e. initial defects appear to normalize [31]. The clinical application are diagnosis of CAD and most importantly, for the assessment of tissue viability, e.g. by radiotracer injection under physical stress with early and delayed imaging protocols, which allows to monitor such redistribution closely over time. Figure 2 shows the superior imaging characteristics of ${ }^{18} \mathrm{~F}$-flurpiridaz PET compared to a common PET MPI agent, ${ }^{13} \mathrm{~N}$-ammonia, in (A) healthy rats and $(\mathrm{B})$ in a rat model after coronary artery occlusion. The ${ }^{18} \mathrm{~F}$-labeled radiotracer demonstrated improved contrast and higher resolution, resulting in better delineation of induced lesions, despite a higher injected dose of ${ }^{13} \mathrm{~N}$-ammonia relative to ${ }^{18} \mathrm{~F}$-flurpiridaz. For the ${ }^{18} \mathrm{~F}$-labeled

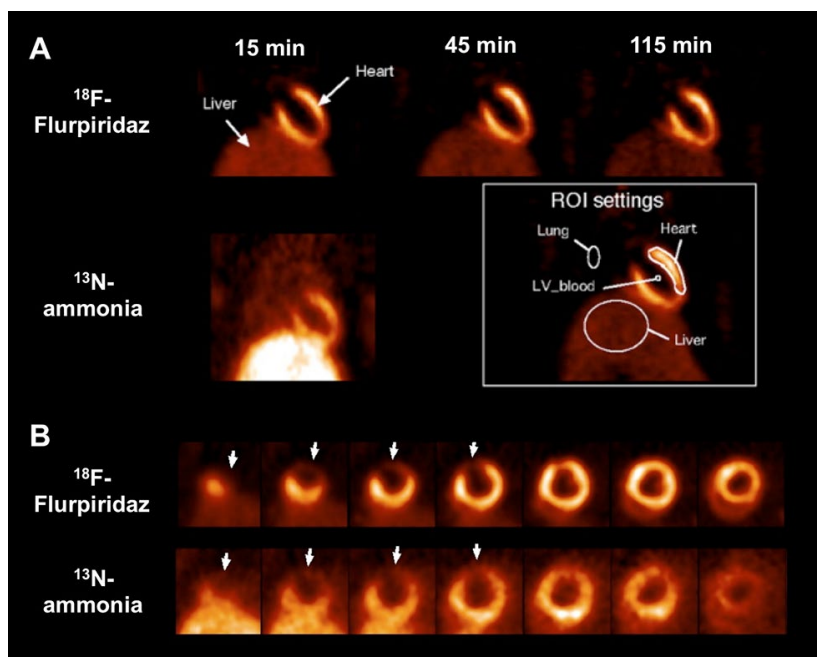

Fig. 2 a Short-axis ${ }^{18}$ F-flurpiridaz PET in a healthy rat at 15,45 and $115 \mathrm{~min}$ post-injection. The left ventricular myocardium showed excellent contrast to surrounding tissues. ${ }^{13} \mathrm{~N}$-ammonia PET at $10 \mathrm{~min}$ in a coronal view. Regions of interest placements are displayed in white box. b Short-axis images of rat hearts 1 week after coronary artery occlusion using ${ }^{18} \mathrm{~F}$-flurpiridaz and ${ }^{13} \mathrm{~N}$-ammonia PET. The induced ${ }^{18} \mathrm{~F}$-flurpiridaz uptake defect visualized at $15 \mathrm{~min}$ corresponded precisely to the defect in ${ }^{13} \mathrm{~N}$-ammonia images. However, ${ }^{18} \mathrm{~F}$-flurpiridaz demonstrated improved contrast and higher resolution resulting in better delineation of induced lesions, despite a higher injected dose of ${ }^{13} \mathrm{~N}$-ammonia $(57 \mathrm{MBq})$ versus ${ }^{18} \mathrm{~F}$-flurpiridaz (37 MBq). The inferior/ left ventricular wall can be better distinguished from the liver due to a more rapid liver clearance of ${ }^{18}$ F-flurpiridaz compared to ${ }^{13} \mathrm{~N}$-ammonia. Modified from Higuchi et al. [32] (C) by the Society of Nuclear Medicine and Molecular Imaging, Inc. 
imaging agent, the inferior/left ventricular wall can be better distinguished from the liver [32]. Figure 3 displays a head-to-head comparison of ${ }^{18} \mathrm{~F}$-flurpiridaz and ${ }^{18} \mathrm{~F}$-FBnTP in a rat model of short-term occlusion and reperfusion. For the latter radiotracer, retention stability over time was confirmed, while ${ }^{18} \mathrm{~F}$-flurpiridaz showed slow restoration over time. Differences may be explained by the underlying uptake mechanisms: ${ }^{18} \mathrm{~F}$-flurpiridaz targets mitochondrial complex I, while ${ }^{18} \mathrm{~F}$-FBnTP localizes to mitochondria due to membrane potential [33]. The observed kinetics (redistribution after reperfusion) may allow for the use of ${ }^{18} \mathrm{~F}$-flurpiridaz in a similar way to clinical protocols for the diagnosis of CAD with conventional stress/rest ${ }^{201} \mathrm{TI}$ perfusion protocols or for the assessment of myocardial viability $[32,34]$. In a permanent and transient occlusion model of the left coronary artery, uptake defect assessed by ${ }^{18} \mathrm{~F}$-flurpiridaz closely correlated with histological measured scar sizes confirmed by 2,3,5-triphenyltetrazolium chloride staining [35]. In a pig model, Guehl et al. demonstrated that accurate rest and stress blood flow estimations with ${ }^{18} \mathrm{~F}$-flurpiridaz are feasible, even in less than 15 min of PET acquisition time by using a singlescan rest-stress method, which further emphasizes the practicality of this radiotracer in clinical routine [36]. Also in a pig model, Sherif et al. showed that ${ }^{18} \mathrm{~F}$-flurpiridaz retention and standardized uptake values (SUVs) correlated with absolute MBF values at rest and pharmacological stress. As such, SUVs may be used as a substitute for absolute blood flow. As SUV does not require determination of radiotracer input function, tracer injection and exercise treadmill or bicycle stress test protocols could be performed outside the scanner. From a practical standpoint, such an approach may facilitate flow estimation in clinical routine [37]. By comparison with radioactive microsphere-derived blood flow in a pig model, a high agreement rate with regional MBF using ${ }^{18} \mathrm{~F}$-flurpiridaz was achieved, even over a wide flow range [38].

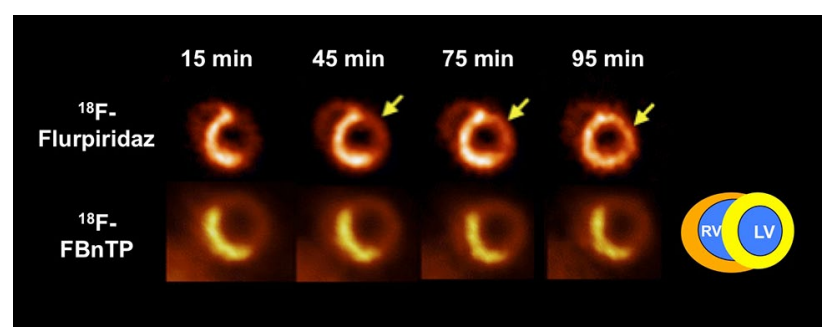

Fig. 3 Head-to-head comparison of both ${ }^{18} \mathrm{~F}$-labeled myocardial perfusion (MPI) PET radiotracers in a rat model of short-term occlusion and reperfusion. Radiotracers $\left[{ }^{18} \mathrm{~F}\right.$-flurpiridaz and ${ }^{18} \mathrm{~F}$-fluorobenzyltriphenyl-phosphonium $\left({ }^{18} \mathrm{~F}\right.$-FBnTP)] were injected during ischemia. ${ }^{18} \mathrm{~F}$-flurpiridaz showed slow restoration of uptake, while ${ }^{18} \mathrm{~F}-\mathrm{FBnTP}$ remained stable over time, i.e. stability and lack of washout was confirmed for ${ }^{18} \mathrm{~F}$-FBnTP [17]. Differences may be explained by different uptake mechanisms of both radiotracers [33]. Modified from Higuchi et al. [32, 33] () by the Society of Nuclear Medicine and Molecular Imaging, Inc

\section{Clinical studies}

In a phase I trial enrolling healthy volunteers, a sustained retention was recorded up to $5 \mathrm{~h}$ post-injection and the radiotracer was well tolerated in all 13 subjects [39]. Clear and homogenous delineation of the myocadium was appreciated up to $5 \mathrm{~h}$ after administration, while liver clearance was observed $2 \mathrm{~h}$ post-injection [39]. Thus, the radiopharmaceutical is present in the myocardium to allow for an administration of the radiotracer at peak treadmill exercise. Moreover, kinetic studies demonstrated that imaging can be performed immediately after completing the exercise protocol and thus, ${ }^{18} \mathrm{~F}$-flurpiridaz may identify even subtle stress-induced wall motion abnormalities (compared to SPECT with ${ }^{99 \mathrm{~m}} \mathrm{Tc}$ agents, which generally involve imaging at least 30 min post-injection) [25]. Apart from that, Packard et al. enrolled seven healthy subjects with a low likelihood of myocardial ischemia and $8 \mathrm{CAD}$ patients using ${ }^{18} \mathrm{~F}$-flurpiridaz. Notably, such a study design provided a wide range of MBF. In patients with no stress-inducible ischemia, no significant differences in MBF (either at rest or adenosine stress) and myocardial flow reserve (MFR) were recorded. This was in contradistinction to CAD subjects: lower MBF in diseased vascular segements after adenosine stress was noted and therefore, also a reduction in MFR [40]. Berman et al. evaluated the efficacy and safety profile of ${ }^{18} \mathrm{~F}$-flurpiridaz in a phase II trial. In 143 subjects from 21 different study sites, stress-rest PET and ${ }^{99 \mathrm{~m}} \mathrm{Tc}$ sestamibi SPECT were performed, while the latter imaging modality served as a comparator. The certainty of interpretation, which was recorded by three blinded readers in a binary fashion (abnormal vs. normal), was considerably higher for PET ( $90.8 \%$ vs. SPECT, 70.9\%). In 86 patients, who also underwent invasive coronary angiography (ICA, as a reference standard for coronary stenosis), PET revealed a higher sensitivity compared to SPECT, while specificity remained similar. Of note, in patients suffering from CAD (detected on invasive procedures), the magnitude of the reversible defect assessed with PET was larger than with SPECT. Figure 4 displays a mismatch of ${ }^{18} \mathrm{~F}$-flurpiridaz PET MPI versus ${ }^{99 \mathrm{~m}} \mathrm{Tc}$-sestamibi SPECT MPI in an 82-year old male with an occluded native proximal left anterior descending (LAD) coronary artery and an occluded left internal mammary graft to the LAD. On ${ }^{18} \mathrm{~F}$-flurpiridaz PET MPI, a reversible perfusion defect throughout the territory of the occluded proximal LAD was noted; the ${ }^{99 \mathrm{~m}} \mathrm{Tc}$-sestamibi images showed only a moderate perfusion defect in the distal LAD territory [41]. Assessing the summed difference score for ${ }^{18} \mathrm{~F}$-flurpiridaz MPI and ${ }^{99 \mathrm{~m}} \mathrm{Tc}$-sestamibi MPI, stress induced perfusion abnormalities in patients with multivessel CAD were significantly higher with PET MPI [42]. Recently, Bateman et al. reported on 795 subjects from 72 international sites and described previous results of the first Phase III trial. The authors 


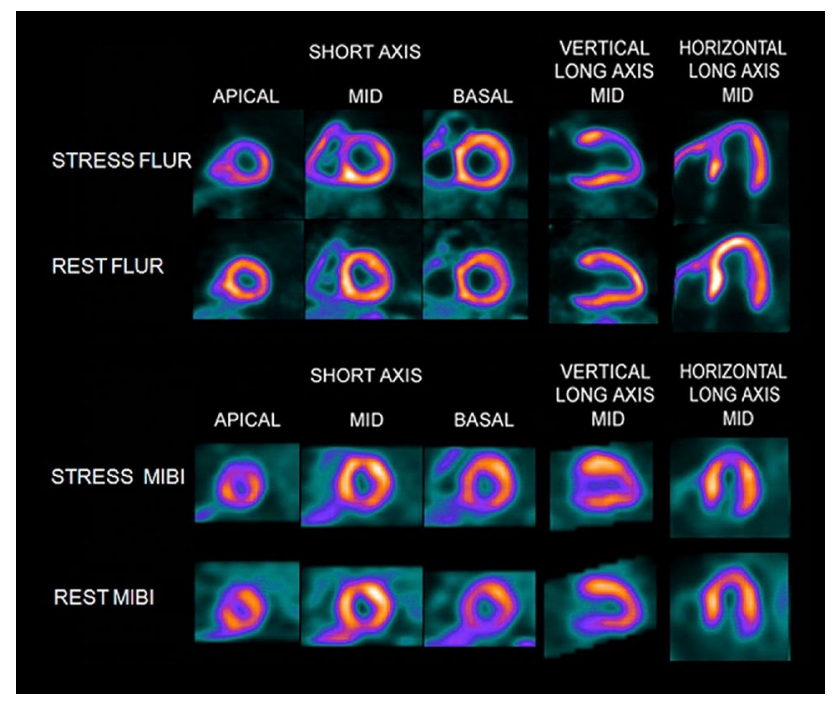

Fig. 4 FLUR PET and MIBI SPECT images from an 82-year-old man. The FLUR PET (top) and MIBI SPECT (bottom) images from an 82-year-old man with shortness of breath and an occluded native proximal left anterior descending (LAD) coronary artery and an occluded left internal mammary graft to the LAD and no other significant native CAD. The FLUR images show a severe reversible perfusion defect throughout the territory of the occluded proximal LAD, whereas the MIBI images show only a moderate perfusion defect in the distal LAD territory (apical slices). FLUR =Flurpiridaz F 18; MIBI $=$ Tc-99m sestamibi. Reprinted from the Journal of the American College of Cardiology (JACC), 61(4), Daniel S. Berman, Jamshid Maddahi, B. K. Tamarappoo, Johannes Czernin, Raymond Taillefer, James E. Udelson, C. Michael Gibson, Marybeth Devine, Joel Lazewatsky, Gajanan Bhat, Dana Washburn, Phase II safety and clinical comparison with single-photon emission computed tomography myocardial perfusion imaging for detection of coronary artery disease: flurpiridaz F 18 positron emission tomography, 469-77, Copyright (2013), with permission from Elsevier [41]

noted superior diagnostic performance characteristics for ${ }^{18} \mathrm{~F}$-flurpiridaz relative to SPECT MPI for the assessment of CAD in obese patients [43]. The recently launched, prospective, international, multi-center, open-label AURORA study (second Phase III study, ClinicalTrials.gov Identifier: NCT03354273) will include subjects with suspected CAD scheduled for ICA and both SPECT and PET MPI will be carried out prior to intervention. The primary endpoint is diagnostic efficacy of ${ }^{18} \mathrm{~F}$-flurpiridaz PET MPI in detecting significant CAD [44, 45].

\section{${ }^{18}$ F-labeled radiotracers for MPI: ${ }^{18} \mathrm{~F}$-FBnTP}

The lipophilic cation ${ }^{18} \mathrm{~F}$-FBnTP also accumulates in myocardial mitochondria [46]. In mongrel dogs, uptake and retention kinetics were tested in vivo and ${ }^{18} \mathrm{~F}-\mathrm{FBnTP}$ reached its plateau in the left ventricle $5 \mathrm{~min}$ after radiotracer administration. A delineation of the myocardium was still seen 90 min post-injection. In addition to that, the metabolite concentration in the blood was considerably low and the heart-to-liver ratio was 1.2 after $60 \mathrm{~min}$. The heart-to-lung ratio was 12:1 (5 min post-injection), which was much higher than reported for ${ }^{99 \mathrm{~m}} \mathrm{Tc}$-labeled SPECT agents (2:1) in the same species. Thus, one may speculate that the lower background activity leads to better imaging contrast relative to SPECT counterparts $[47,48]$. In addition, ${ }^{18} \mathrm{~F}$-FBnTP was also compared to ${ }^{99 \mathrm{~m}} \mathrm{Tc}$-tetrofosmin SPECT in vivo by using various degrees of coronary artery stenosis: 17 dogs with different degrees of stenosis of LAD or circumflex coronary arteries were enrolled. Microsphere flow was assessed with radioactive micropsheres, which allow for distinction of true myocardial blood flow in ischemic versus non-ischemic beds of the left ventricle. Compared to ${ }^{99 \mathrm{~m}} \mathrm{Tc}$-tetrofosmin, superior diagnostic performance for ${ }^{18} \mathrm{~F}$-FBnTP was reported, in particular for the assessment of mild or severe stenosis [49]. To reveal further insights into kinetics of ${ }^{18} \mathrm{~F}$-FBnTP, short transient coronary artery occlusion (ligation of the left coronary artery, $2 \mathrm{~min}$ ) was induced in Wistar rats, which was followed by reperfusion. PET imaging with ${ }^{18} \mathrm{~F}-\mathrm{FBnTP}$ showed that the radiotracer remained stable demonstrating no washout or redistribution and matched histologically proven defect areas [33]. Recently, in a rat model of autoimmune myocarditis, the longitudinal imaging characteristics of ${ }^{18} \mathrm{~F}$-FDG were investigated and ${ }^{18} \mathrm{~F}$-FBnTP was used as a reference perfusion marker [50]. Albeit this radiotracer is used in a preclinical setting over the last years, human data are still lacking and thus, if a more widespread adoption is envisaged, further clinical trials are warranted. In addition to ${ }^{18} \mathrm{~F}$-FBnTP, 18F-labeled fluoroalkylphosphonium derivatives $\left({ }^{18} \mathrm{~F}\right.$-FATPs) have been synthesized as well: these are $\left(5-{ }^{18} \mathrm{~F}\right.$-fluoropentyl)triphenylphosphonium cation $\left({ }^{18} \mathrm{~F}-\mathrm{FPTP}\right),\left(6-{ }^{18} \mathrm{~F}\right.$-fluorohexyl $)$ triphenylphosphonium cation $\left({ }^{18} \mathrm{~F}-\mathrm{FHTP}\right)$, and $\left(2-\left(2-{ }^{18} \mathrm{~F}\right.\right.$-fluoroethoxy) ethyl)triphenylphosphonium cation $\left({ }^{18} \mathrm{~F}\right.$-FETP). Compared with ${ }^{13} \mathrm{~N}$-ammonia in a rat model of coronary occlusion, ${ }^{18}$ F-FATPs showed excellent image quality, along with rapid liver and lung clearance [51].

Table 1 summarizes key properties of ${ }^{18} \mathrm{~F}$-labeled radiotracers for PET MPI. Supplementary Table 1 displays characteristics of established PET MPI agents and the novel PET agent ${ }^{18} \mathrm{~F}$-flurpiridaz.

\section{Future directions}

${ }^{18}$ F-labeled radiotracers allow for an improved targetto-background ratio compared to commonly used PET MPI agents, which in turn leads to higher imaging quality [32]. Thus, given the superior imaging characteristics of ${ }^{18} \mathrm{~F}$-labeled PET MPI radiotracers compared to other SPECT or PET MPI competitors, it is possible that these novel radiotracers further contribute to an even more 
Table 1 Advantages and limitations of the reviewed ${ }^{18} \mathrm{~F}$-labeled PET radiotracers for MPI, namely ${ }^{18} \mathrm{~F}$-flurpiridaz and ${ }^{18} \mathrm{~F}$-fluorobenzyltriphenylphosphonium $\left({ }^{18} \mathrm{~F}-\mathrm{FBnTP}\right)$

\begin{tabular}{|c|c|c|}
\hline${ }^{18} \mathrm{~F}$ MPI PET radiotracers & Advantages & Limitations \\
\hline${ }^{18} \mathrm{~F}$-flurpiridaz & $\begin{array}{l}\text { - Considerable high first-pass extraction of }>90 \% \\
\text { [27, 28] } \\
\text { - Almost linear correlation between tracer uptake } \\
\text { and cardiac blood flow in isolated perfused rat } \\
\text { hearts [28] } \\
\text { - Radiotracer redistribution after reperfusion, } \\
\text { i.e. }{ }^{18} \text { F-flurpiridaz may be suitable for clinical } \\
\text { protocols similar to conventional stress/rest }{ }^{201} \mathrm{TI} \\
\text { perfusion protocols or assessment of myocardial } \\
\text { viability [32] } \\
\text { - Phase I: radiotracer present up to } 5 \text { h post- } \\
\text { injection, i.e. injection at peak treadmill exercise } \\
\text { is feasible [39] } \\
\text { - Phase II: compared to stress-rest SPECT MPI, } \\
\text { PET MPI with superior performance characteris- } \\
\text { tics for overall CAD diagnosis [41] } \\
\text { - First Phase III study: superior perfusion defect } \\
\text { detection of }{ }^{18} \text { F-flurpiridaz relative to SPECT } \\
\text { MPI for the assessment of CAD in obese sub- } \\
\text { jects [43] } \\
\text { - Second Phase III study (AURORA): will assess } \\
\text { the efficacy of }{ }^{18} \text { F-flurpiridaz PET MPI in } \\
\text { detecting significant CAD compared to SPECT } \\
\text { MPI in patients scheduled for invasive coronary } \\
\text { angiography [44, 45] }\end{array}$ & $\begin{array}{l}\text { - Limited to academic } \\
\text { centers } \\
\text { - Cyclotron production } \\
\text { - No FDA approval yet } \\
\text { - Cost-effectiveness } \\
\text { data are lacking }\end{array}$ \\
\hline${ }^{18} \mathrm{~F}-\mathrm{FBnTP}$ & $\begin{array}{l}\text { - Superior diagnostic performance for }{ }^{18} \mathrm{~F}-\mathrm{FBnTP} \\
\text { compared to SPECT MPI in dogs [49] } \\
\text { - Lack of redistribution in a rat model of short } \\
\text { transient coronary artery occlusion ( } 2 \text { min) and } \\
\text { reperfusion (i.e. tracer injection remote from the } \\
\text { imaging device may be feasible, e.g. in a chest } \\
\text { pain unit) [33] }\end{array}$ & $\begin{array}{l}\text { - No larger clinical } \\
\text { trials } \\
\text { - Limited to academic } \\
\text { centers } \\
\text { - Cyclotron production } \\
\text { - Cost-effectiveness } \\
\text { data are lacking }\end{array}$ \\
\hline
\end{tabular}

SPECT single photon emission tomography, CAD coronary artery disease, FDA Food and Drug Administration tailored treatment approach for ischemic heart patients. Notably, the extraction fraction of those radiotracers at various flow rates open the door for optimal absolute MBF quantification [40]. Cutoff values of both MBF and MFR could be established with those radiotracers and thus, could be used for risk stratification [40]. This would apply to different subgroups in a clinical context which are at higher risk of cardiac events, such as diabetes or chronic kidney diseases [52]. The latter group is of great interest, as cardiovascular disease is the main cause of death among patients suffering from severe renal dysfunction [53]. However, conventional SPECT MPI cannot identify high-risk patients across a wide spectrum of renal (mal) function and thus, novel approaches using ${ }^{18} \mathrm{~F}$ PET MPI radiotracers may have an increased prognostic capability [54]. In addition, quantification of MBF (assessed by ${ }^{82} \mathrm{Rb}$ PET) in patients prior to heart transplantation can also identify subjects at high risk of suffering from later clinical events [55]. However, the longer half-life of ${ }^{18} \mathrm{~F}$ PET MPI along with their superior imaging quality may allow for a more practical adoption in clinical routine and a more thorough evaluation of the perfusion status in heart transplant recipients. Other applications of such radiotracers include chest pain with normal findings on coronary angiography [52]. In a similar vein like for MPI PET agents, a recent shift from established cardiac neuronal PET agents ( ${ }^{11} \mathrm{C}$-hydroxyephedrine) towards novel ${ }^{18} \mathrm{~F}$-labeled PET tracers to measure cardiac nerve integrity has been noted, e.g. by the use of the myocardial nerve imaging agent ${ }^{18} \mathrm{~F}$ LMI1195 [56]. Thus, in a dual-tracer approach, both newly introduced ${ }^{18} \mathrm{~F}$ radiotracers $\left({ }^{18} \mathrm{~F}\right.$-flurpiridaz for $\mathrm{MBF}$ and ${ }^{18}$ F-LMI1195 for cardiac nerve integrity) could be used. Such a global functional assessment of the heart has been also previously tested in a rat model of ischemia (with ${ }^{11} \mathrm{C}-\mathrm{HED}$ and ${ }^{201} \mathrm{TI}$ for perfusion): compared to the perfusion defect areas, a larger ${ }^{11} \mathrm{C}-\mathrm{HED}$ uptake defect in both subacute and chronic phases was noted [57]. Thus, further clinical applications, preferably with ${ }^{18} \mathrm{~F}$-labeled cardiac perfusion/nerve tracers, which offer superior imaging quality, would be of great interest. 


\section{Conclusions}

${ }^{18} \mathrm{~F}$-labeled radionuclides for PET MPI perform well in assessing the defect size in CAD patients. First, they are less expensive to produce and may also be distributed using delivery systems from central cyclotron facilities [23]. Second, the longer half-life of ${ }^{18} \mathrm{~F}$-labeled MPI agents also allow for delayed imaging protocols, which in turn may allow for physical exercise stress testing protocols outside of the scanner [17]. In light of its excellent extraction fraction, ${ }^{18} \mathrm{~F}$-flurpiridaz has very favorable characteristics as a PET MPI agent and phase II/III trials have reported on a superior diagnostic performance relative to common SPECT MPI agents [39, 41, 43]. In the currently recruiting AURORA trial, subjects referred for ICA because of suspected CAD will undergo both SPECT and PET MPI prior to intervention $[44,45]$. The results may reveal further insights into the efficacy of ${ }^{18}$ F-flurpiridaz PET MPI in detecting significant CAD. However, MPI (either with PET or SPECT) still remains underrepresented in some countries: for instance, in Germany, CAD diagnosis seems to be mainly shifted directly to invasive angiographic procedures, which in turn leads to less requests of such tests in clinical routine [58].

Funding This work was supported by the Competence Network of Heart Failure funded by the Integrated Research and Treatment Center (IFB) of the Federal Ministry of Education and Research (BMBF) and German Research Council (DFG Grant HI 1789/3-3 and CH 1516/2-1). This project has received funding from the European Union's Horizon 2020 research and innovation programme under the Marie SklodowskaCurie Grant Agreement No. 701983.

\section{Compliance with ethical standards}

Conflict of interest The authors declare that they have no conflict of interest.

Research involving human participants or animals This article does not contain any studies with human participants or animals performed by any of the authors.

Open Access This article is distributed under the terms of the Creative Commons Attribution 4.0 International License (http://creativeco mmons.org/licenses/by/4.0/), which permits unrestricted use, distribution, and reproduction in any medium, provided you give appropriate credit to the original author(s) and the source, provide a link to the Creative Commons license, and indicate if changes were made.

\section{References}

1. Heidenreich PA, Albert NM, Allen LA, Bluemke DA, Butler J, Fonarow GC, Ikonomidis JS, Khavjou O, Konstam MA, Maddox TM, Nichol G, Pham M, Pina IL, Trogdon JG, American Heart Association Advocacy Coordinating C, Council on Arteriosclerosis T, Vascular B, Council on Cardiovascular R, Intervention,
Council on Clinical C, Council on E, Prevention, Stroke C (2013) Forecasting the impact of heart failure in the United States: a policy statement from the American Heart Association. Circ Heart Fail 6 (3):606-619. https://doi.org/10.1161/HHF.0b013e3182 91329a

2. Dunlay SM, Roger VL (2014) Understanding the epidemic of heart failure: past, present, and future. Curr Heart Fail Rep 11(4):404-415. https://doi.org/10.1007/s11897-014-0220-X

3. Luscher TF (2018) Heart failure subgroups: HFrEF, HFmrEF, and HFpEF with or without mitral regurgitation. Eur Heart $\mathbf{J}$ 39(1):1-4. https://doi.org/10.1093/eurheartj/ehx750

4. Ponikowski P, Voors AA, Anker SD, Bueno H, Cleland JGF, Coats AJS, Falk V, Gonzalez-Juanatey JR, Harjola VP, Jankowska EA, Jessup M, Linde C, Nihoyannopoulos P, Parissis JT, Pieske B, Riley JP, Rosano GMC, Ruilope LM, Ruschitzka F, Rutten FH, van der Meer P, Group ESCSD (2016) 2016 ESC Guidelines for the diagnosis and treatment of acute and chronic heart failure: the Task Force for the diagnosis and treatment of acute and chronic heart failure of the European Society of Cardiology (ESC)Developed with the special contribution of the Heart Failure Association (HFA) of the ESC. Eur Heart J 37(27):2129-2200. https:// doi.org/10.1093/eurheartj/ehw128

5. Delepaul B, Robin G, Delmas C, Moine T, Blanc A, Fournier P, Roger-Rolle A, Domain G, Delon C, Uzan C, Boudjellil R, Carrie D, Roncalli J, Galinier M, Lairez O (2017) Who are patients classified within the new terminology of heart failure from the 2016 ESC guidelines? ESC Heart Fail 4(2):99-104. https://doi. org/10.1002/ehf2.12131

6. Lala A, Desai AS (2014) The role of coronary artery disease in heart failure. Heart Fail Clin 10(2):353-365. https://doi. org/10.1016/j.hfc.2013.10.002

7. Greenland P, Gaziano JM (2003) Clinical practice. Selecting asymptomatic patients for coronary computed tomography or electrocardiographic exercise testing. N Engl J Med 349(5):465473. https://doi.org/10.1056/NEJMcp023197

8. Verberne HJ, Acampa W, Anagnostopoulos C, Ballinger J, Bengel F, De Bondt P, Buechel RR, Cuocolo A, van Eck-Smit BL, Flotats A, Hacker M, Hindorf C, Kaufmann PA, Lindner O, Ljungberg M, Lonsdale M, Manrique A, Minarik D, Scholte AJ, Slart RH, Tragardh E, de Wit TC, Hesse B, European Association of Nuclear M (2015) EANM procedural guidelines for radionuclide myocardial perfusion imaging with SPECT and SPECT/CT: 2015 revision. Eur J Nucl Med Mol Imaging 42(12):1929-1940. https ://doi.org/10.1007/s00259-015-3139-x

9. Rowe SP, Gorin MA, Pomper MG (2017) Imaging of prostatespecific membrane antigen using [(18)F]DCFPyL. PET Clin 12(3):289-296. https://doi.org/10.1016/j.cpet.2017.02.006

10. Werner RA, Bluemel C, Allen-Auerbach MS, Higuchi T, Herrmann K (2015) 68Gallium- and 90Yttrium-/177Lutetium: "theranostic twins" for diagnosis and treatment of NETs. Ann Nucl Med 29(1):1-7. https://doi.org/10.1007/s12149-014-0898-6

11. Lertsburapa K, Ahlberg AW, Bateman TM, Katten D, Volker L, Cullom SJ, Heller GV (2008) Independent and incremental prognostic value of left ventricular ejection fraction determined by stress gated rubidium 82 PET imaging in patients with known or suspected coronary artery disease. J Nucl Cardiol 15(6):745-753. https://doi.org/10.1007/BF03007355

12. Bateman TM (2004) Cardiac positron emission tomography and the role of adenosine pharmacologic stress. Am J Cardiol 94(2A): 19D-24D (discussion 24D-25D). https://doi. org/10.1016/j.amjcard.2004.04.013

13. Werner RA, Wakabayashi H, Chen X, Hirano M, Shinaji T, Lapa C, Rowe SP, Javadi MS, Higuchi T (2018) Functional renal imaging with 2-deoxy-2-(18)F-fluorosorbitol PET in rat models of renal disorders. J Nucl Med 59(5):828-832. https://doi. org/10.2967/jnumed.117.203828 
14. Driessen RS, Raijmakers PG, Stuijfzand WJ, Knaapen P (2017) Myocardial perfusion imaging with PET. Int J Cardiovasc Imaging 33(7):1021-1031. https://doi.org/10.1007/s1055 4-017-1084-4

15. Schindler TH, Quercioli A, Valenta I, Ambrosio G, Wahl RL, Dilsizian V (2014) Quantitative assessment of myocardial blood flow-clinical and research applications. Semin Nucl Med 44(4):274-293. https://doi.org/10.1053/j.semnuclmed 2014.04.002

16. Schindler TH (2016) Myocardial blood flow: putting it into clinical perspective. J Nucl Cardiol 23(5):1056-1071. https://doi. org/10.1007/s12350-015-0372-4

17. Rischpler C, Park MJ, Fung GS, Javadi M, Tsui BM, Higuchi $\mathrm{T}$ (2012) Advances in PET myocardial perfusion imaging: F-18 labeled tracers. Ann Nucl Med 26(1):1-6. https://doi.org/10.1007/ s12149-011-0552-5

18. Nakazato R, Berman DS, Alexanderson E, Slomka P (2013) Myocardial perfusion imaging with PET. Imaging Med 5(1):35-46. https://doi.org/10.2217/iim.13.1

19. Li Y, Zhang W, Wu H, Liu G (2014) Advanced tracers in PET imaging of cardiovascular disease. Biomed Res Int 2014:504532. https://doi.org/10.1155/2014/504532

20. Kaufmann PA, Gnecchi-Ruscone T, Yap JT, Rimoldi O, Camici PG (1999) Assessment of the reproducibility of baseline and hyperemic myocardial blood flow measurements with 150-labeled water and PET. J Nucl Med 40(11):1848-1856

21. Rauch B, Helus F, Grunze M, Braunwell E, Mall G, Hasselbach W, Kubler W (1985) Kinetics of 13N-ammonia uptake in myocardial single cells indicating potential limitations in its applicability as a marker of myocardial blood flow. Circulation 71(2):387-393

22. Kobayashi R, Chen X, Werner RA, Lapa C, Javadi MS, Higuchi T (2017) New horizons in cardiac innervation imaging: introduction of novel (18)F-labeled PET tracers. Eur J Nucl Med Mol Imaging 44(13):2302-2309. https://doi.org/10.1007/s00259-017-3828-8

23. Ducharme J, Goertzen AL, Patterson J, Demeter S (2009) Practical aspects of 18F-FDG PET when receiving 18F-FDG from a distant supplier. J Nucl Med Technol 37(3):164-169. https://doi. org/10.2967/jnmt.109.062950

24. Werner RA, Rischpler C, Onthank D, Lapa C, Robinson S, Samnick S, Javadi M, Schwaiger M, Nekolla SG, Higuchi T (2015) Retention kinetics of the 18F-labeled sympathetic nerve PET tracer LMI1195: comparison with 11C-hydroxyephedrine and 123I-MIBG. J Nucl Med 56(9):1429-1433. https://doi. org/10.2967/jnumed.115.158493

25. Maddahi J, Packard RR (2014) Cardiac PET perfusion tracers: current status and future directions. Semin Nucl Med 44(5):333343. https://doi.org/10.1053/j.semnuclmed.2014.06.011

26. Hirano M, Werner RA, Higuchi T (2018) A New era of myocardial perfusion imaging: 18F PET Tracer. ANC. https://doi. org/10.17996/anc.18-00056

27. Schuler F, Casida JE (2001) The insecticide target in the PSST subunit of complex I. Pest Manag Sci 57(10):932-940. https://doi. org/10.1002/ps.364

28. Huisman MC, Higuchi T, Reder S, Nekolla SG, Poethko T, Wester HJ, Ziegler SI, Casebier DS, Robinson SP, Schwaiger M (2008) Initial characterization of an 18F-labeled myocardial perfusion tracer. J Nucl Med 49(4):630-636. https://doi.org/10.2967/jnume d.107.044727

29. Yu M, Guaraldi MT, Mistry M, Kagan M, McDonald JL, Drew K, Radeke H, Azure M, Purohit A, Casebier DS, Robinson SP (2007) BMS-747158-02: a novel PET myocardial perfusion imaging agent. J Nucl Cardiol 14(6):789-798. https://doi.org/10.1016/j. nuclcard.2007.07.008

30. Yu M, Bozek J, Guaraldi M, Kagan M, Azure M, Robinson SP (2010) Cardiac imaging and safety evaluation of BMS747158, a novel PET myocardial perfusion imaging agent, in chronic myocardial compromised rabbits. J Nucl Cardiol 17(4):631-636. https://doi.org/10.1007/s12350-010-9221-7

31. Pagnanelli RA, Basso DA (2010) Myocardial perfusion imaging with 201Tl. J Nucl Med Technol 38(1):1-3. https://doi. org/10.2967/jnmt. 109.068593

32. Higuchi T, Nekolla SG, Huisman MM, Reder S, Poethko T, Yu M, Wester HJ, Casebier DS, Robinson SP, Botnar RM, Schwaiger M (2008) A new 18F-labeled myocardial PET tracer: myocardial uptake after permanent and transient coronary occlusion in rats. J Nucl Med 49(10):1715-1722. https://doi.org/10.2967/jnume d.108.053967

33. Higuchi T, Fukushima K, Rischpler C, Isoda T, Javadi MS, Ravert H, Holt DP, Dannals RF, Madar I, Bengel FM (2011) Stable delineation of the ischemic area by the PET perfusion tracer 18F-fluorobenzyl triphenyl phosphonium after transient coronary occlusion. J Nucl Med 52(6):965-969. https://doi.org/10.2967/ jnumed.110.085993

34. Rischpler C, Higuchi T, Fukushima K, Javadi MS, Merrill J, Nekolla SG, Bravo PE, Bengel FM (2012) Transient ischemic dilation ratio in $82 \mathrm{Rb}$ PET myocardial perfusion imaging: normal values and significance as a diagnostic and prognostic marker. J Nucl Med 53(5):723-730. https://doi.org/10.2967/jnume d.111.097600

35. Sherif HM, Saraste A, Weidl E, Weber AW, Higuchi T, Reder S, Poethko T, Henriksen G, Casebier D, Robinson S, Wester HJ, Nekolla SG, Schwaiger M (2009) Evaluation of a novel (18) F-labeled positron-emission tomography perfusion tracer for the assessment of myocardial infarct size in rats. Circ Cardiovasc Imaging 2(2):77-84. https://doi.org/10.1161/CIRCIMAGIN G.108.815423

36. Guehl NJ, Normandin MD, Wooten DW, Rozen G, Sitek A, Ruskin J, Shoup TM, Ptaszek LM, El Fakhri G, Alpert NM (2017) Single-scan rest/stress imaging: validation in a porcine model with (18)F-Flurpiridaz. Eur J Nucl Med Mol Imaging 44(9):15381546. https://doi.org/10.1007/s00259-017-3684-6

37. Sherif HM, Nekolla SG, Saraste A, Reder S, Yu M, Robinson S, Schwaiger M (2011) Simplified quantification of myocardial flow reserve with flurpiridaz F 18: validation with microspheres in a pig model. J Nucl Med 52(4):617-624. https://doi.org/10.2967/ jnumed.110.083196

38. Nekolla SG, Reder S, Saraste A, Higuchi T, Dzewas G, Preissel A, Huisman M, Poethko T, Schuster T, Yu M, Robinson S, Casebier D, Henke J, Wester HJ, Schwaiger M (2009) Evaluation of the novel myocardial perfusion positron-emission tomography tracer 18F-BMS-747158-02: comparison to $13 \mathrm{~N}$-ammonia and validation with microspheres in a pig model. Circulation 119(17):23332342. https://doi.org/10.1161/CIRCULATIONAHA.108.797761

39. Maddahi J, Czernin J, Lazewatsky J, Huang SC, Dahlbom M, Schelbert H, Sparks R, Ehlgen A, Crane P, Zhu Q, Devine M, Phelps M (2011) Phase I, first-in-human study of BMS747158, a novel $18 \mathrm{~F}$-labeled tracer for myocardial perfusion PET: dosimetry, biodistribution, safety, and imaging characteristics after a single injection at rest. J Nucl Med 52(9):1490-1498. https://doi. org/10.2967/jnumed.111.092528

40. Packard RR, Huang SC, Dahlbom M, Czernin J, Maddahi J (2014) Absolute quantitation of myocardial blood flow in human subjects with or without myocardial ischemia using dynamic flurpiridaz F 18 PET. J Nucl Med 55(9):1438-1444. https://doi.org/10.2967/ jnumed.114.141093

41. Berman DS, Maddahi J, Tamarappoo BK, Czernin J, Taillefer R, Udelson JE, Gibson CM, Devine M, Lazewatsky J, Bhat G, Washburn D (2013) Phase II safety and clinical comparison with single-photon emission computed tomography myocardial perfusion imaging for detection of coronary artery disease: flurpiridaz $\mathrm{F}$ 18 positron emission tomography. J Am Coll Cardiol 61(4):469477. https://doi.org/10.1016/j.jacc.2012.11.022 
42. Maddahi J, Czernin J, Berman D, Taillefer R, Devine M, Lazewatsky J, Bhat G, Washburn D (2011) Comparison of flurpiridaz F 18 PET injection and Tc-99m labeled SPECT myocardial perfusion imaging for identifying severity and extent of stress induced myocardial ischemia in phase 2 clinical trials. J Nucl Med 52(no:supplement 1):444

43. Bateman TM, Maddahi J, Udelson J, Beanlands R, Knuuti J, Heller G, Berman D, Lazewatsky J, Orlandi C (2016) Improved assessment of cad in obese subjects with flurpiridaz F18 PET myocardial perfusion imaging: a subset analysis of the flurpiridaz F18 301 phase 3 study. JACC 67(13 Supplement): 1578

44. https://www.medicaldevice-network.com/news/ge-healthcare-initi ates-new-phase-iii-trial-flurpiridaz-detect-cad/. Last downloaded August 4, 2018

45. https://clinicaltrials.gov/ct2/show/study/NCT03354273-contacts. An international study to evaluate diagnostic efficacy of flurpiridaz (18F) injection PET MPI in the detection of coronary artery disease (CAD) last downloaded August 5, 2018

46. Madar I, Ravert H, Nelkin B, Abro M, Pomper M, Dannals R, Frost JJ (2007) Characterization of membrane potential-dependent uptake of the novel PET tracer 18F-fluorobenzyl triphenylphosphonium cation. Eur J Nucl Med Mol Imaging 34(12):2057-2065. https://doi.org/10.1007/s00259-007-0500-8

47. Madar I, Ravert HT, Du Y, Hilton J, Volokh L, Dannals RF, Frost JJ, Hare JM (2006) Characterization of uptake of the new PET imaging compound $18 \mathrm{~F}$-fluorobenzyl triphenyl phosphonium in dog myocardium. J Nucl Med 47(8):1359-1366

48. Sinusas AJ, Shi Q, Saltzberg MT, Vitols P, Jain D, Wackers FJ, Zaret BL (1994) Technetium-99m-tetrofosmin to assess myocardial blood flow: experimental validation in an intact canine model of ischemia. J Nucl Med 35(4):664-671

49. Madar I, Ravert H, Dipaula A, Du Y, Dannals RF, Becker L (2007) Assessment of severity of coronary artery stenosis in a canine model using the PET agent 18F-fluorobenzyl triphenyl phosphonium: comparison with $99 \mathrm{mTc}$-tetrofosmin. J Nucl Med 48(6):1021-1030. https://doi.org/10.2967/jnumed.106.038778

50. Werner RA, Wakabayashi H, Bauer J, Schütz C, Zechmeister C, Hayakawa N, Javadi MS, Lapa C, Jahns R, Süleyman E, Jahns V, Higuchi T (2018) Longitudinal 18F-FDG PET imaging in a rat model of autoimmune myocarditis. Eur Heart J Cardiovasc Imaging. https://doi.org/10.1093/ehjci/jey119

51. Kim DY, Kim HS, Reder S, Zheng JH, Herz M, Higuchi T, Pyo AY, Bom HS, Schwaiger M, Min JJ (2015) Comparison of 18F-labeled fluoroalkylphosphonium cations with $13 \mathrm{~N}-\mathrm{NH} 3$ for PET myocardial perfusion imaging. J Nucl Med 56(10):15811586. https://doi.org/10.2967/jnumed.115.156794
52. Murthy VL, Bateman TM, Beanlands RS, Berman DS, BorgesNeto S, Chareonthaitawee P, Cerqueira MD, deKemp RA, DePuey EG, Dilsizian V, Dorbala S, Ficaro EP, Garcia EV, Gewirtz H, Heller GV, Lewin HC, Malhotra S, Mann A, Ruddy TD, Schindler TH, Schwartz RG, Slomka PJ, Soman P, Di Carli MF, Directors SCCBo, Directors ABo (2018) Clinical quantification of myocardial blood flow using PET: joint position paper of the SNMMI cardiovascular council and the ASNC. J Nucl Med 59 (2):273-293. https://doi.org/10.2967/jnumed.117.201368

53. Collins AJ, Foley RN, Chavers B, Gilbertson D, Herzog C, Johansen K, Kasiske B, Kutner N, Liu J, St Peter W, Guo H, Gustafson S, Heubner B, Lamb K, Li S, Li S, Peng Y, Qiu Y, Roberts T, Skeans M, Snyder J, Solid C, Thompson B, Wang C, Weinhandl E, Zaun D, Arko C, Chen SC, Daniels F, Ebben J, Frazier E, Hanzlik C, Johnson R, Sheets D, Wang X, Forrest B, Constantini E, Everson S, Eggers P, Agodoa L (2012) 'United States Renal Data System 2011 Annual Data Report: Atlas of chronic kidney disease \& end-stage renal disease in the United States. Am J Kidney Dis 59(1 Suppl 1):A7. https://doi.org/10.1053/j.ajkd.2011.11.015e1 -420 .

54. Al-Mallah MH, Hachamovitch R, Dorbala S, Di Carli MF (2009) Incremental prognostic value of myocardial perfusion imaging in patients referred to stress single-photon emission computed tomography with renal dysfunction. Circ Cardiovasc Imaging 2(6):429-436. https://doi.org/10.1161/CIRCIMAGIN G.108.831164

55. Mc Ardle BA, Davies RA, Chen L, Small GR, Ruddy TD, Dwivedi G, Yam Y, Haddad H, Mielniczuk LM, Stadnick E, Hessian R, Guo A, Beanlands RS, deKemp RA, Chow BJ (2014) Prognostic value of rubidium- 82 positron emission tomography in patients after heart transplant. Circ Cardiovasc Imaging 7(6):930 937. https://doi.org/10.1161/CIRCIMAGING.114.002184

56. Werner RA, Chen X, Hirano M, Rowe SP, Lapa C, Javadi M, Higuchi T (2018) SPECT vs. PET in cardiac innervation imaging: clash of the titans. Clin Transl Imaging 6(4):293-303

57. Werner RA, Maya Y, Rischpler C, Javadi MS, Fukushima K, Lapa C, Herrmann K, Higuchi T (2016) Sympathetic nerve damage and restoration after ischemia-reperfusion injury as assessed by (11) C-hydroxyephedrine. Eur J Nucl Med Mol Imaging 43(2):312318. https://doi.org/10.1007/s00259-015-3171-x

58. Lindner O, Burchert W, Schafer W, Hacker M (2017) Myocardial perfusion SPECT 2015 in Germany. Results of the 7(th) survey. Nuklearmedizin 56(1):31-38. https://doi.org/10.3413/Nukme d-0858-16-10 